\title{
La autoeficacia y su influencia en la insatisfacción de la imagen corporal: revisión sistemática
}

\author{
Self-efficacy and its influence \\ in the dissatisfaction of body image: Systematic review
}

\author{
Nayalis Nápoles Neyra, Humberto Blanco Vega \\ y Perla Jannet Jurado García \\ Universidad Autónoma de Chihuahua ${ }^{1}$
}

Autor para correspondencia: Perla Jannet Jurado García,pjurado@uach.mx.

\section{RESUMEN}

\begin{abstract}
Introducción: La autoeficacia influye de forma efectiva en la imagen corporal de los adolescentes. Objetivo: Analizar por medio de una revisión sistemática la relación entre autoeficacia y la insatisfacción de la imagen corporal. Método: Se realizó una búsqueda en las bases de datos Scopus, Scielo, Dialnet, Redalyc, Ebsco y Pubmed. Resultados: Inicialmente se identificaron 479 documentos, de los cuales 25 cumplieron con los criterios de inclusión. Discusión: Mediante la revisión efectuada, se confirma que la autoeficacia es una variable mediadora entre el estilo de vida saludable en cuanto a la alimentación y el ejercicio físico y las percepciones corporales y la satisfacción con la imagen corporal.
\end{abstract}

Palabras clave: Revisión sistemática; Insatisfacción de la imagen corporal; Autoeficacia; Adolescentes.

\begin{abstract}
Introduction: Self-efficacy affects adolescents'body image. Objective: To explore the relationship between self-efficacy and dissatisfaction with body image in adolescents through a systematic review of the research literature. Method: A search of databases, such as Scopus, Scielo, Dialnet, Redalyc, Ebsco, and Pubmed, was conducted. Results: even though 479 documents were identified for initial revision, only 25 fulfilled the inclusion criteria. Discussion: As expected, self-efficacy acts as one mediational variable between a healthy lifestyle regarding nutrition, physical exercise, body perceptions, and satisfaction with body image.
\end{abstract}

Key words: Systematic review; Dissatisfaction of body image; Self-efficacy; Adolescents.

\footnotetext{
${ }^{1}$ Doctorado en Cultura Física, Paseos de Chihuahua, 31125 Chihuahua, Chih., México, tel. (614)158-99-00, correos electrónicos: nnapolesn2@ gmail.com, hblanco@uach.mx y pjurado@uach.mx.
} 
Recibido: $19 / 05 / 2020$

Aceptado: 17/08/2020

\section{Autoeficacia}

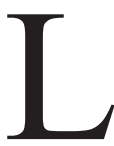

a autoeficacia es entendida como el juicio que cada persona tiene sobre sus capacidades para enfrentar o ejecutar acciones que les permita obtener un resultado (Blanco, Díaz, Ornelas y Mondaca, 2019; Moreno y Bueno, 2019). Fue descrita por Bandura (1977) como la médula de la teoría cognitivo- social y definida como la influencia que puede tener un individuo sobre lo que hace a través de su pensamiento reflexivo y del uso de sus habilidades y destrezas para logar una conducta concreta. Para una mejor comprensión de la misma, se debe tener en cuenta la interrelación de la persona, su conducta y el ambiente, en la que dicha triada, a partir de los conocimientos, emociones e influencias ambientales, junto al comportamiento, operan de forma interactiva (Gutiérrez, 2016).

La autoeficacia se ha vinculado a comportamientos saludables, como la regulación del peso (Silva, Fernández y Rodríguez, 2019), y de igual modo al consumo de frutas y verduras (Pallo, 2017). Algunos estudios han revelado que la autoeficacia es un elemento principal en el proceso de transformación de conductas saludables al prever la capacidad de las personas para hacer dieta (Quiliano, 2015). Tal capacidad permite que los individuos sean más dinámicos, por lo que llevan a cabo actividades físicas para mejorar su salud (Fraile, Tejero, Esteban y Veiga, 2019).

En México se han llevado a cabo algunos estudios que utilizan la autoeficacia como un factor rector de la actividad física (Herencia, 2017; Olivos, 2018) o de conductas saludables (Pérez et al., 2018; Estrada, Campero, Suárez, De la Vara y González, 2017; Orcasita, Mosquera y Carrillo, 2018). Así pues, la autoeficacia es una emoción de valor individual, de aprecio y eficacia en la conducción de la vida, y se puede calcular a través del resultado de la actividad realizada, la experiencia, los estados fisiológicos y la persuasión. Así, una autoeficacia baja puede causar efectos negativos en la calidad de vida; por ejemplo, cuando se relaciona con la imagen corporal, las personas que tienen una imagen corporal positiva alcanzan una autoeficacia alta y tienen confianza en sí mismas. Además, debe destacarse que en la teoría cognitiva social, la autoeficacia da importancia a acciones educativas cuyo fin es reducir la angustia generada por una imagen corporal no deseada y aumentar así la autoestima (Ghahremani, Hemmati, Kaveh y Fararoei, 2018).

\section{Imagen corporal}

La imagen corporal es un constructo que se ha convertido en una inquietud esencial para muchas personas, especialmente para los jóvenes, quienes gastan mucho tiempo y dinero para cambiar la apariencia de su cuerpo (Pruzinsky y Cash, 1990). Se define como la representación de un ideal con el que una persona contrasta su apariencia física; que la autoimagen sea positiva o negativa puede incidir en todos los aspectos de su comportamiento, y también puede estar influida por el crecimiento físico, los accidentes y las lesiones (Ghahremani et al., 2018; Gillen y Lefkowitz, 2012). Asimismo, también se considera que la imagen corporal es la percepción que se establece mentalmente del propio cuerpo y está integrada por elementos biológicos, psicológicos y sociales, como la edad, el sexo, el índice de masa corporal (IMC), la satisfacción o insatisfacción, el estatus socioeconómico y otros, e influenciada por la interacción social, la familia, la cultura y los medios de comunicación (León, González, Fernández y Contreras, 2018).

De igual manera, la relación multidisciplinar de la imagen corporal puede establecerse desde el punto de vista sociológico, el cual concibe el cuerpo como un medio de comunicación entre el individuo y el mundo; en cuanto al aspecto psicológico y su relación con el autoconcepto existe una correlación positiva con el bienestar psicológico y negativa con el malestar psicológico. El aspecto educativo es de gran importancia para la formación del yo como identidad personal del educando, en el que intervienen valores, ideales, cosmovisión y cultura, entre otros. La disciplina de la educación física define la imagen corporal como una entidad psicosomática en la que interviene la relación entre mente y cuerpo. Por tanto, multidimensionalmente el constructo comprende 
desde las emociones y creencias sobre el propio cuerpo, hasta las autorrepresentaciones, efectos y actividades referentes al mismo (Castro, 2017).

La imagen corporal está estrechamente relacionada con la obesidad (López, Díaz y Smith, 2018) y con diversos factores sociales y del contexto sociocultural enfocados en la apariencia física (Arévalo, 2017; Pacheco, Lozano y González, 2018), la violencia física y psicológica (Calvo, Pimiento y Ríos, 2019; Da Silva y Hasselmann, 2019; Reyna, 2019), el bienestar subjetivo y la satisfacción con la vida (Fraile et al., 2019; Venegas, 2019). De igual modo, otras investigaciones la han vinculado a las conductas alimentarias (Cano y Gianella, 2019; Díaz, Bilbao, Unikel, Escalante y Parra, 2019), el sexo y el estado nutricional (Cruzat, Díaz, Lizana y Castro, 2016; Delgado et al., 2017), así como al IMC, la motivación, la autocompasión y la autoestima (De La Cruz, Arévalo y Rojas, 2018; Fernández, González, Contreras y Cuevas, 2015; Horcajo, Quiles y Quiles, 2019; Pérez y Trujillo, 2018; Sierra, 2017). En resumen, la imagen corporal guarda una estrecha relación con las personas, así como sus intereses, aspiraciones, motivaciones e ideales respecto a su apariencia física y a la satisfacción con su cuerpo (Babativa, 2016).

La imagen corporal es, pues, un constructo que se define como un sentimiento subjetivo sobre la propia apariencia, la cual tiene dos facetas: una imagen corporal positiva y una negativa, las que desempeñan un papel esencial en la formación de la identidad individual, de modo que una imagen corporal positiva facilita el funcionamiento psicosocial, mientras que una negativa conduce a la insatisfacción y a una baja autoestima (Yadav, 2017).

\section{Insatisfacción con la imagen corporal}

Muchas personas están descontentas con alguna parte de su cuerpo, lo que si bien no altera necesariamente las acciones que realizan de forma cotidiana, sí representa un malestar; no obstante, este desata comportamientos perjudiciales para la salud cuando la persona pretende cambiar su apariencia, lo que se ha denominado insatisfacción corporal. (Ojeda, 2016). La insatisfacción corporal puede definirse como la percepción negativa sobre el cuerpo, y puede estar asociada con una

Instituto de Investigaciones Psicológicas - Universidad Veracruzana ISSN impreso: 1405-1109 baja eficacia ya que los individuos manifiestan intranquilidad respecto a su aspecto físico y a su evaluación social (Gómez, Gutierrez, Llanos y Babativa, 2017).

En la literatura científica, la insatisfacción con la imagen corporal también se señala como una experiencia negativa del individuo sobre sí mismo, sin la cual la persona no sentiría la necesidad de cambiar su cuerpo llevando a cabo actividades o comportamientos con consecuencias negativas (Solano, Cano, Blanco y Fernández, 2017).

La distorsión perceptiva es originada por la insatisfacción corporal debido a la diferencia entre la representación ideal y la observada, es decir, por la preocupación hacia el propio cuerpo que puede establecerse por el sexo, la edad, la sexualidad, la clase social o los grupos psicológicos, debido a factores sociales como los medios de comunicación, las amistades, la familia, las emociones, las creencias y, en general, la cultura (Jiménez, Cruz y Bacardí, 2017).

En las investigaciones que han abordado la insatisfacción corporal se le ha relacionado con una alimentación desordenada (Manrique et al., 2018), intentos de suicidio (Guadarrama, Carrillo, Márquez, Hernández y Veytia, 2014) y pobre calidad de vida (Bonilla, Romero y Cabrera, 2015).

Varios estudios han mostrado un nivel alto de insatisfacción con imagen corporal entre los adolescentes, principalmente del sexo femenino, quienes realizan actividades no saludables para reducir su peso (Da Silva y Hasselmann, 2019). Como es bien sabido, existe una representación de un cuerpo ideal perfecto en la actualidad, y cuando no se logra se pueden crear perturbaciones de la imagen corporal, además de los efectos en la salud y en el comportamiento de los jóvenes, toda vez que este grupo etario se encuentra en un proceso de crecimiento, lo que puede provocar en ellos enfermedades y trastornos diversos (Bazán y Miño, 2015). Así pues, la problemática que resulta de lo anterior es la insatisfacción corporal alta ya que el cuerpo ideal es generado por los medios de comunicación -en especial por las redes sociales-, por lo que los adolescentes se convierten en un grupo de riesgo en el que se pueden manifestar conductas inapropiadas que pueden complicar la salud de los mismos (Venegas y González, 2020). 
Dado lo anterior, es necesario efectuar acciones educativas de intervención en la etapa de la adolescencia mediante las cuales se corrija la autopercepción de la imagen corporal negativa-la que se relaciona estrechamente con los trastornos alimenticios-, incluyendo en ellas a la familia y la escuela como elementos transformadores de la persona (De Pinho et al., 2019).

\section{Influencia de la imagen corporal en los adolescentes}

Como se ha mencionado, la adolescencia es un periodo de crecientes cambios morfológicos y psicosociales que inciden considerablemente en la apreciación que los jóvenes tienen del cuerpo. En ellos, el deseo y la búsqueda constante de un físico ideal se han vuelto más comunes, lo que puede repercutir en una grave distorsión de su imagen corporal (De Pinho et al., 2019). Además, la imagen corporal está relacionada con el bienestar subjetivo, y ambos factores influyen durante el proceso de la adolescencia de un modo tal que pueden contribuir a tener una imagen propia deformada o inadecuada, así como a una evaluación corporal subjetiva negativa de la apariencia física con consecuencias psicológicas, esto es, a la insatisfacción corporal (Molina, Gonçalves, Guimaraes y Aerts, 2018).

En consecuencia, es importante conocer los estilos de vida de los adolescentes porque son la vía que utilizan para conformar patrones de conductas determinados, en cuanto que en esta etapa de la vida es la que las relaciones interpersonales prevalecen sobre la adquisición de hábitos. No se puede dejar de mencionar que en este periodo se modifica su marco social a través de su inserción en la educación superior, donde pueden adquirir otras rutinas y prácticas inapropiadas, como el consumo de alcohol, tabaco y otras drogas; el cambio en su patrón de alimentación, el sedentarismo o la promiscuidad, todo lo cual desemboca en una salud física y psicológica inadecuada (Molina et al., 2018; Moral, Agraso, Ramos, Jiménez y Jiménez, 2020).

Cabe señalar que los estudios realizados han corroborado que la adolescencia es un grupo en riesgo de padecer obesidad, por lo que es sensible al desarrollo de una insatisfacción causada por las modificaciones biológicas que ocurren en el cuerpo, y a ejecutar actividades no saludables como una vía de resolución de problemas. Por ello, es prudente poner en efecto estrategias con el fin de promover estilos de vida con dietas saludables y ejercicios físicos diarios para mantener un estado nutricional adecuado, así como acciones psicológicas que generen una autoestima alta y la percepción de una correcta imagen corporal (Duno y Acosta, 2019).

\section{Influencia de la autoeficacia en la insatisfacción corporal}

De acuerdo con Simón (2017), existen factores como el autoconcepto, la autoestima, la salud general y la depresión que tienen un efecto positivo o negativo sobre la imagen corporal. Si esta se ve afectada, puede ocasionar trastornos como la diminución del apetito, anorexia nerviosa, bulimia, cambios anormales de peso, trastornos mentales y del sueño, entre otros, todos ellos problemas de salud.

De hecho, la autoestima y la percepción corporal comienzan en la mente de la persona, no en el ideal que se construye; depende de cómo se autovalore y se estime; si no le agrada su cuerpo o alguna parte de su cuerpo, es difícil que se sienta bien consigo misma como un todo.

Por lo anteriormente dicho, el objetivo del presente estudio fue analizar la influencia que existe entre autoeficacia e insatisfacción de la imagen corporal en adolescentes mediante la revisión de estudios publicados sobre este tópico.

\section{MÉTODO}

\section{Muestra}

Se llevó a cabo una revisión sistemática en las siguientes bases de datos electrónicas: Scientific Electronic Library Online (Scielo), Scopus, Pubmed, Dialnet, Redalyc y Ebsco, como estrategia para compilar diversas investigaciones efectuadas en el periodo de enero de 2015 a marzo de 2020, para analizar la relación entre la autoeficacia y la insatisfacción de la imagen corporal en los adoles- 
centes entre las edades de 8 a 19 años, de ambos sexos. Se utilizaron para ello las siguientes palabras clave en español, inglés y portugués: autoeficacia, insatisfacción de la imagen corporal, imagen corporal, adolescentes; self-efficacy, dissatisfaction of body image, body image, teenagers; auto-eficácia, insatisfacao da imagen corporal, imagen corporal y adolescentes. Se conjugaron con el descriptor booleano and.

Como criterios de inclusión se consideraron los artículos originales y de investigaciones indizadas. Se desecharon todos aquellos artículos que no tuvieran relación con las palabras clave, y además los relacionados con enfermedades con cualquier diagnóstico gastrointestinal, enfermedades del corazón, asma bronquial, trastornos de infecciones e inflamatorios, embarazo y consumo del alcohol, y que el contenido no reflejara dicha relación o que no cumpliera con el objetivo del presente estudio.

\section{Extracción de datos}

La información obtenida de la exploración de los artículos se dividió en tres tópicos:

Datos generales del artículo: Base de datos en las que se encontraba, título, autor(es), nombre de la revista, año de publicación, país y palabras clave.

Población de referencia: Rangos de edad, tamaño de la muestra.

Variables utilizadas: "autoeficacia", "insatisfacción de la imagen corporal" y "adolescentes".

Después del filtrado de la búsqueda, se seleccionaron 25 artículos que cumplían con los criterios de inclusión, además de interés para el tema abordado. En la Figura 1 se muestra el diagrama de flujo de la búsqueda bibliográfica.

Figura 1. Diagrama de flujo de la búsqueda bibliográfica.
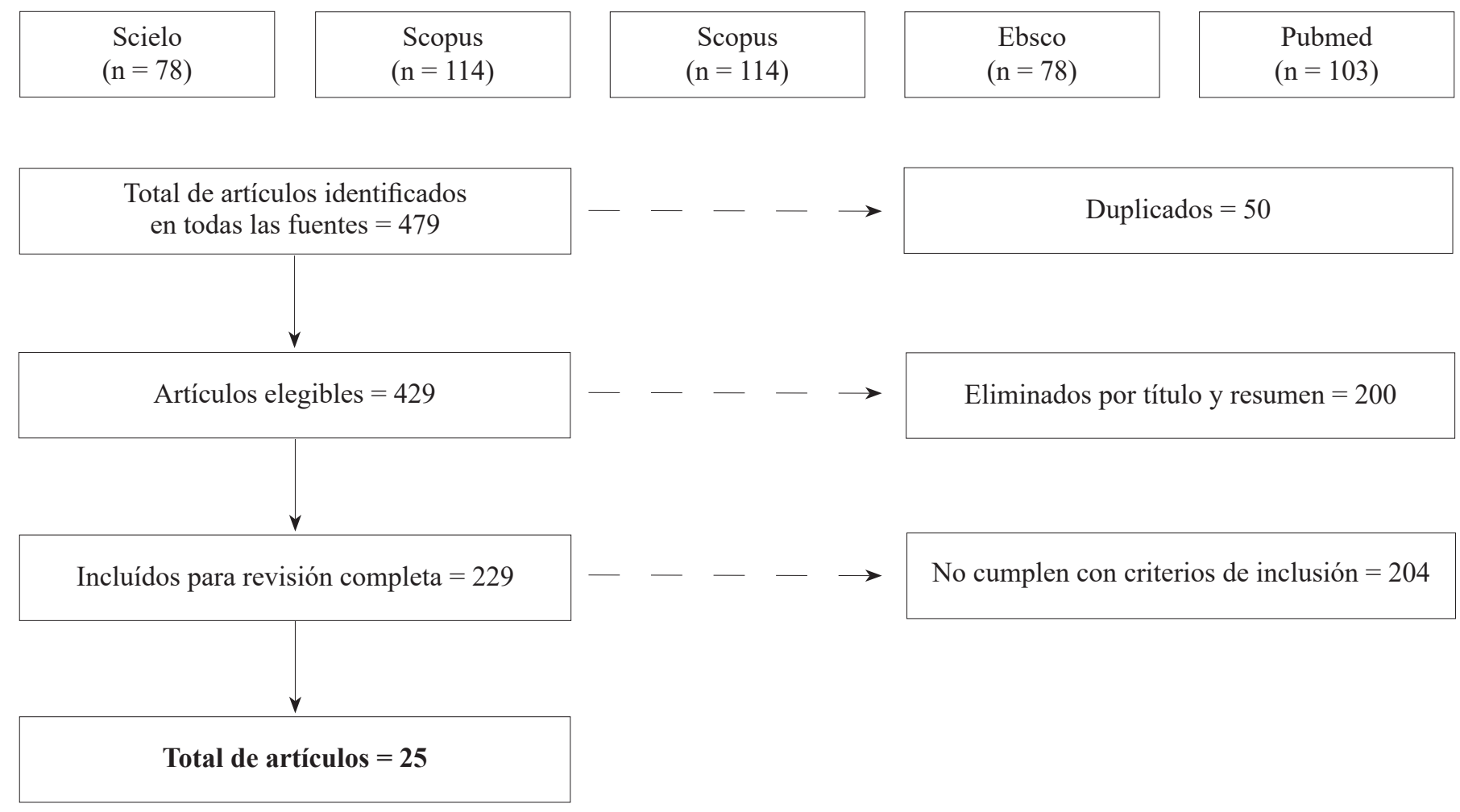

\section{RESULTADOS}

En un primer momento de la búsqueda se identificó un total de 479 artículos, de los cuales se eliminaron

Instituto de Investigaciones Psicológicas - Universidad Veracruzana ISSN impreso: 1405-1109
50 por estar duplicados, quedando como artículos elegibles 429. Se descartaron 200 por título y resumen, y se incluyeron para la revisión completa del texto 229, de los que se excluyeron 204 por no 
cumplir con los criterios, quedando para la presente revisión los 25 artículos que se muestran en la Tabla 1.
Para el estudio de los documentos escogidos se construyó una tabla registro con los siguientes datos: autor, año, país, tamaño de la muestra, instrumentos y resultados (Tabla 1).

Tabla 1. Registro de artículos de estudio relativos a la autoeficacia y la insatisfacción corporal.

\begin{tabular}{|c|c|c|c|c|}
\hline Autor(es) & País & Muestra & Instrumento & Resultados \\
\hline $\begin{array}{l}\text { Duno } \\
\text { y Acosta, } \\
2019 .\end{array}$ & Venezuela & $\begin{array}{l}\mathrm{n}=143 \\
\mathrm{H}(\mathrm{n}=26) \\
\mathrm{M}(\mathrm{n}=117)\end{array}$ & $\begin{array}{l}\text { Test de Siluetas de Gramerd. } \\
\text { Estrato socioeconómico. } \\
\text { Test Godin Shepard } \\
\text { Condición física general (CF). }\end{array}$ & $\begin{array}{l}\text { Elevado porcentaje de insatisfac- } \\
\text { ción corporal en ambos sexos: } \\
84.6 \% \text { (mujeres) y } 80.4 \% \\
\text { (hombres). }\end{array}$ \\
\hline $\begin{array}{l}\text { Escolar et al., } \\
2019 .\end{array}$ & España & $\begin{array}{l}\mathrm{n}=561 \\
\mathrm{H}(\mathrm{n}=240) \\
\mathrm{M}(\mathrm{n}=321)\end{array}$ & $\begin{array}{l}\text { Inventario de Trastornos de la } \\
\text { Conducta Alimentaria.Cuestiona- } \\
\text { rio de Remisión (EDI-3-RF. } \\
\text { Datos demográficos (sexo, edad, } \\
\text { grado, residencia). }\end{array}$ & $\begin{array}{l}\text { Presencia significativa de insatisfac- } \\
\text { ción corporal, más en las mujeres } \\
(2 \%) \text { que en los varones }(0.7 \%) \text {. }\end{array}$ \\
\hline Ramírez, 2017. & España & $\begin{array}{l}\mathrm{n}=1430 \\
\mathrm{H}(\mathrm{n}=836) \\
\mathrm{M}(\mathrm{n}=594)\end{array}$ & $\begin{array}{l}\text { Imagen Corporal Percibida. } \\
\text { Imagen Corporal Ideal. } \\
\text { Imagen Corporal Social. }\end{array}$ & Baja satisfacción corporal. \\
\hline $\begin{array}{l}\text { Peralta, Emelin } \\
\text { y Quintana, } \\
2016 .\end{array}$ & Perú & $\mathrm{N}=108$ & $\begin{array}{l}\text { Entrevistas. } \\
\text { Porcentaje de percepción equivo- } \\
\text { cada de peso o talla. Percepción } \\
\text { sobre imagen corporal y prácticas } \\
\text { para corregirla. }\end{array}$ & $\begin{array}{l}36.1 \% \text { tenían percepción equivoca- } \\
\text { da de su peso y } 50.9 \% \text { de su talla. } \\
\text { En la fase cualitativa tenían una } \\
\text { percepción equivocada del peso. }\end{array}$ \\
\hline $\begin{array}{l}\text { Bully, Elosua } \\
\text { y Jáuregui, } \\
2016 .\end{array}$ & España & $\begin{array}{l}\mathrm{n}=4314 \\
\mathrm{H}(\mathrm{n}=2161) \\
\mathrm{M}(\mathrm{n}=2153)\end{array}$ & $\begin{array}{l}\text { Escala de Insatisfacción } \\
\text { Corporal. }\end{array}$ & $\begin{array}{l}\text { Se observó una disminución no } \\
\text { significativa de los valores medios } \\
\text { de insatisfacción corporal en la } \\
\text { muestra de mujeres. }\end{array}$ \\
\hline $\begin{array}{l}\text { De Pinho et al., } \\
2019 .\end{array}$ & Brasil & $\mathrm{n}=535$ & $\begin{array}{l}\text { Cuestionario sociodemográfico e } \\
\text { imagen corporal. }\end{array}$ & $\begin{array}{l}\text { Los adolescentes muestran insatis- } \\
\text { facción con su imagen corporal. }\end{array}$ \\
\hline $\begin{array}{l}\text { Flanagan } \\
\text { y Perry, } 2018 .\end{array}$ & Estados Unidos & $\mathrm{n}=28$ & $\begin{array}{l}\text { Encuesta Collins Body Figure } \\
\text { Perception. } \\
\text { Encuestas evaluando la insatis- } \\
\text { facción corporal, la eficacia del } \\
\text { ejercicio y la la PFP. } \\
\text { Medidas de aptitud física. }\end{array}$ & $\begin{array}{l}\text { Relación significativa entre insatis- } \\
\text { facción corporal y autopercepción } \\
\text { de aptitud física. }\end{array}$ \\
\hline $\begin{array}{l}\text { Moffitt, } \\
\text { Neumann y } \\
\text { Williamson, } \\
2018 .\end{array}$ & Australia & $\mathrm{n}=149$ & $\begin{array}{l}\text { Escala de Autocompasión. Escala } \\
\text { de Autoestima de Rosenberg. } \\
\text { Subescala de Insatisfacción Cor- } \\
\text { poral del Inventario de Trastornos } \\
\text { Alimenticios (EDI-BD), Escalas } \\
\text { analógicas visuales (VAS). }\end{array}$ & $\begin{array}{l}\text { La insatisfacción con el peso y con } \\
\text { la apariencia fueron significativa- } \\
\text { mente más bajas en el grupo de } \\
\text { autocompasión que en los grupos } \\
\text { de autoestima y control. y la moti- } \\
\text { vación de superación personal fue } \\
\text { significativamente mayor. }\end{array}$ \\
\hline $\begin{array}{l}\text { Hernández } \\
\text { et al., } 2016 .\end{array}$ & México & $\begin{array}{l}\mathrm{n}=282 \\
\mathrm{H}(\mathrm{n}=38) \\
\mathrm{M}(\mathrm{n}=144)\end{array}$ & $\begin{array}{l}\text { Cuestionario de Imagen } \\
\text { Corporal. } \\
\text { Test Infantil de Actitudes } \\
\text { Alimentarias. } \\
\text { Cuestionario de Actitudes } \\
\text { Socioculturales hacia } \\
\text { la Apariencia. }\end{array}$ & $\begin{array}{l}\text { La interiorización del ideal corporal } \\
\text { es el principal predictor de la insa- } \\
\text { tisfacción corporal en ambos sexos. }\end{array}$ \\
\hline
\end{tabular}

Continúa... 


\begin{tabular}{|c|c|c|c|c|}
\hline $\begin{array}{l}\text { Caldera et al., } \\
2019 .\end{array}$ & México & $\begin{array}{l}\mathrm{n}=567 \\
\mathrm{H}(\mathrm{n}=279) \\
\mathrm{M}(\mathrm{n}=288)\end{array}$ & $\begin{array}{l}\text { Cuestionario de Insatisfacción } \\
\text { con la Imagen Corporal } \\
\text { (Body Shape Questionnaire) } \\
\text { Inventario de Personalidad NEO. }\end{array}$ & $\begin{array}{l}\text { Se identificaron variables predic- } \\
\text { toras de la imagen corporal insa- } \\
\text { tisfecha. Se encontraron relaciones } \\
\text { significativas entre las siguientes } \\
\text { dimensiones de la personalidad: } \\
\text { neuroticismo, extroversión, ama- } \\
\text { bilidad y responsabilidad con la } \\
\text { imagen corporal. }\end{array}$ \\
\hline $\begin{array}{l}\text { Verdugo, } \\
\text { Armas, Baños, } \\
\text { Moncada y } \\
\text { Rentería, } 2020 .\end{array}$ & $\begin{array}{l}\text { México. } \\
\text { Costa Rica }\end{array}$ & $\mathrm{n}=56$ & $\begin{array}{l}\text { Escala de Factores de Riesgo } \\
\text { de Trastornos Alimentarios } \\
\text { (EFRATA). }\end{array}$ & $\begin{array}{l}\text { Los gimnastas varones exhibieron } \\
\text { un mayor grado de insatisfacción } \\
\text { corporal al desear una silueta más } \\
\text { gruesa o musculosa, mientras que } \\
\text { las mujeres aspiraban a ser más } \\
\text { delgadas respecto al grupo control. }\end{array}$ \\
\hline $\begin{array}{l}\text { Platas, Gómez } \\
\text { y Pineda, } 2019 .\end{array}$ & México & $\begin{array}{l}\mathrm{n}=549 \\
\mathrm{H}(\mathrm{n}=284) \\
\mathrm{M}(\mathrm{n}=265)\end{array}$ & $\begin{array}{l}\text { Batería de pruebas con propieda- } \\
\text { des psicométricas. }\end{array}$ & $\begin{array}{l}\text { La totalidad de la muestra se encon- } \\
\text { traba insatisfecha y con alteración } \\
\text { de la imagen corporal; específ- } \\
\text { camente, subestimaban su peso } \\
\text { corporal (tamaño y forma). }\end{array}$ \\
\hline $\begin{array}{l}\text { Venegas y } \\
\text { González, } \\
2020 \text {. }\end{array}$ & México & $\begin{array}{l}\mathrm{n}=206 \\
\mathrm{M}(\mathrm{n}=206)\end{array}$ & $\begin{array}{l}\text { Cuestionario de la Figura Corpo- } \\
\text { ral y Cuestionario de Influencias } \\
\text { del Modelo Estético Corporal. }\end{array}$ & $\begin{array}{l}\text { Los modelos propuestos son } \\
\text { evidencia de que las variables } \\
\text { socioculturales influyen de manera } \\
\text { significativa en la creación de una } \\
\text { imagen corporal negativa. }\end{array}$ \\
\hline $\begin{array}{l}\text { Franco, Díaz y } \\
\text { Bautista, } 2019 .\end{array}$ & México & $\begin{array}{l}\mathrm{n}=487 \\
\mathrm{M}(\mathrm{n}=487)\end{array}$ & $\begin{array}{l}\text { Test de Actitudes Alimentarias. } \\
\text { Cuestionario de Imagen } \\
\text { Corporal. }\end{array}$ & $\begin{array}{l}18.8 \% \text { de las adolescentes y } 19.8 \% \\
\text { de las jóvenes manifestaron insatis- } \\
\text { facción corporal. }\end{array}$ \\
\hline $\begin{array}{l}\text { Mendoza y } \\
\text { Olalde, } 2019 .\end{array}$ & México & $\mathrm{n}=187$ & $\begin{array}{l}\text { Cuestionario Breve de Conductas } \\
\text { Alimentarias de Riesgo Sfs-test. }\end{array}$ & $\begin{array}{l}\text { Se observó una insatisfacción } \\
\text { severa en } 26.7 \% \text {. Las mujeres con } \\
\text { sobrepeso tenían más probabilida- } \\
\text { des de estar insatisfechas con su } \\
\text { imagen corporal. }\end{array}$ \\
\hline $\begin{array}{l}\text { Albertson, Neff } \\
\text { y Dill, } 2015 .\end{array}$ & Brasil & $\mathrm{n}=201$ & $\begin{array}{l}\text { Body Area Scale. } \\
\text { Escala de Violencia Psicológica. } \\
\text { Conflict Tactics Scales, Form R } \\
\text { (CTS-1). }\end{array}$ & $\begin{array}{l}\text { La violencia psicológica está } \\
\text { asociada a la insatisfacción de la } \\
\text { imagen corporal solamente en ado- } \\
\text { lescentes de sexo femenino. }\end{array}$ \\
\hline $\begin{array}{l}\text { Sánchez, } \\
\text { López, Sgroi y } \\
\text { Díaz, } 2019 .\end{array}$ & Italia & $\begin{array}{l}\mathrm{n}=335 \\
\mathrm{H}(\mathrm{n}=198) \\
\mathrm{M}(\mathrm{n}=137)\end{array}$ & Siluetas de Stunkard. & $\begin{array}{l}\text { El problema psicológico de la } \\
\text { insatisfacción corporal tuvo una } \\
\text { mayor prevalencia que el problema } \\
\text { fisiológico de la obesidad. }\end{array}$ \\
\hline $\begin{array}{l}\text { Amaya, } \\
\text { Alvarez, } \\
\text { Ortega } \\
\text { y Mancilla, } \\
2019 .\end{array}$ & México & $\begin{array}{l}\mathrm{n}=175 \\
\mathrm{H}(\mathrm{n}=80) \\
\mathrm{M}(\mathrm{n}=95)\end{array}$ & $\begin{array}{l}\text { Test Infantil de Actitudes Ali- } \\
\text { mentarias (CHEAT). Cuestionario } \\
\text { de Imagen Corporal (BSQ-16). } \\
\text { Cuestionario de Actitudes So- } \\
\text { cioculturales hacia la Apariencia } \\
\text { (versión revisada (SATAQ-R). } \\
\text { Inventario de Influencia de Pares } \\
\text { sobre la Preocupación Alimenta- } \\
\text { ria (I-PIEC). }\end{array}$ & $\begin{array}{l}\text { La asociación de la influencia de } \\
\text { pares y la interiorización del ideal } \\
\text { corporal con la insatisfacción cor- } \\
\text { poral fueron más altas que con las } \\
\text { conductas alimentarias anómalas; } \\
\text { por el contrario, el IMC fue una de } \\
\text { las variables con las correlacio- } \\
\text { nes más bajas con insatisfacción } \\
\text { corporal y conductas alimentarias } \\
\text { anómalas. }\end{array}$ \\
\hline
\end{tabular}




\begin{tabular}{|c|c|c|c|c|}
\hline Vurgun, 2015. & Turquía & $\begin{array}{l}\mathrm{n}=45 \\
\mathrm{H}(\mathrm{n}=20) \\
\mathrm{M}(\mathrm{n}=25)\end{array}$ & $\begin{array}{l}\text { Cuestionario de Satisfacción } \\
\text { de Imagen Corporal, Escala de } \\
\text { Self-efficacy. }\end{array}$ & $\begin{array}{l}\text { Las mujeres que hacen ejercicio } \\
\text { están más satisfechas con su imagen } \\
\text { corporal, en comparación con muje- } \\
\text { res sedentarias. El IMC fue encon- } \\
\text { trado como el predictor más fuerte } \\
\text { de satisfacción corporal. }\end{array}$ \\
\hline $\begin{array}{l}\text { Rostami, } \\
\text { Fallahi, } \\
\text { Pashaei } \\
\text { y Roshani, } \\
2017 .\end{array}$ & Irán & $\begin{array}{l}\mathrm{n}=816 \\
\mathrm{M}(\mathrm{n}=816)\end{array}$ & $\begin{array}{l}\text { Cuestionario (información } \\
\text { demográfica). } \\
\text { Construcciones de modelos } \\
\text { transteóricos. } \\
\text { Evaluación de imágenes } \\
\text { corporales. }\end{array}$ & $\begin{array}{l}\text { El efecto de interacción } \\
\text { de la autoeficacia y la imagen } \\
\text { corporal sobre el comportamiento } \\
\text { del ejercicio fue estadísticamente } \\
\text { significativo } \\
(p<0.05) .\end{array}$ \\
\hline $\begin{array}{l}\text { Molina et al., } \\
2018 .\end{array}$ & Brasil & $\mathrm{n}=1460$ & $\begin{array}{l}\text { Cuestionario sociodemográfico. } \\
\text { Criterios de Clasificación Econó- } \\
\text { mica de Brasil. } \\
\text { Cuestionario de Forma Corporal } \\
\text { Escala de Satisfacción de Vida de } \\
\text { los Estudiantes. } \\
\text { Medidas multidimensionales de } \\
\text { felicidad y percepción de salud. }\end{array}$ & $\begin{array}{l}\text { La mayoría de los participantes } \\
\text { estaban satisfechos con su imagen } \\
\text { corporal. }\end{array}$ \\
\hline $\begin{array}{l}\text { Palacios } \\
\text { y Ramírez, } \\
2016 .\end{array}$ & México & $\begin{array}{l}\mathrm{n}=1012 \\
\mathrm{H}(\mathrm{n}=531) \\
\mathrm{M}(\mathrm{n}=481)\end{array}$ & $\begin{array}{l}\text { Conductas alimentarias. Subes- } \\
\text { cala del Inventario de Palacios } \\
\text { (2015) sobre autoeficacia. }\end{array}$ & $\begin{array}{l}\text { Las mujeres se preocupan más por } \\
\text { su peso, en comparación con los } \\
\text { hombres. }\end{array}$ \\
\hline $\begin{array}{l}\text { Ghahremani } \\
\text { et al., } 2018 .\end{array}$ & Irán & $\begin{array}{l}\mathrm{n}=150 \\
(72 \text { en el } \\
\text { grupo de } \\
\text { intervención } \\
\text { y } 78 \text { en el } \\
\text { grupo de } \\
\text { control) }\end{array}$ & $\begin{array}{l}\text { Preocupación por la imagen cor- } \\
\text { poral de Littleto. Cuestionarios } \\
\text { de Autoestima de Rosenberg. }\end{array}$ & $\begin{array}{l}\text { Disminución significativa del nivel } \\
\text { de preocupaciones sobre la ima- } \\
\text { gen corporal en los estudiantes del } \\
\text { grupo experimental, en compara- } \\
\text { ción con el grupo de control, y un } \\
\text { aumento de la autoestima de los } \\
\text { estudiantes en el grupo experimen- } \\
\text { tal, en comparación con el grupo } \\
\text { control. }\end{array}$ \\
\hline $\begin{array}{l}\text { Rossi, Trevisol, } \\
\text { Dos Santos, } \\
\text { Dapieve } \\
\text { y Von, } 2020 .\end{array}$ & Brasil & $\begin{array}{l}\mathrm{n}=296 \\
\mathrm{H}(\mathrm{n}=127) \\
\mathrm{M}(\mathrm{n}=169)\end{array}$ & $\begin{array}{l}\text { Ficha de datos } \\
\text { sociodemográficos. } \\
\text { Escala de Autoeficacia } \\
\text { General Percibida (EAGP). } \\
\text { Escala de Motivación para } \\
\text { Aprender (EMA-EM). }\end{array}$ & $\begin{array}{l}\text { Correlación positiva entre } \\
\text { la autoeficacia y la motivación } \\
\text { intrínseca, una correlación negativa } \\
\text { entre la autoeficacia y la motiva- } \\
\text { ción extrínseca, medias más altas } \\
\text { para la motivación intrínseca en las } \\
\text { mujeres y la motivación extrínseca } \\
\text { en los hombres, y medias más altas } \\
\text { en autoeficacia en los hombres. }\end{array}$ \\
\hline $\begin{array}{l}\text { Meza } \\
\text { y Pompa, } \\
2018 .\end{array}$ & México & $\begin{array}{l}\mathrm{n}=540 \\
\mathrm{H}(\mathrm{n}=132) \\
\mathrm{M}(\mathrm{n}=408)\end{array}$ & $\begin{array}{l}\text { Datos antropométricos } \\
\text { (peso, talla, IMC). } \\
\text { Autopercepción de imagen } \\
\text { corporal actual e ideal. }\end{array}$ & $\begin{array}{l}\text { Diferencia entre la imagen corporal } \\
\text { real e ideal, y también entre la ima- } \\
\text { gen real y el grupo de IMC. Además, } \\
\text { los resultados revelan que a mayor } \\
\text { IMC, mayor distorsión de la imagen } \\
\text { corporal y mayor insatisfacción con } \\
\text { la imagen en mujeres. }\end{array}$ \\
\hline
\end{tabular}

Nota: IMC (Índice de Masa Corporal), n (muestra), M (mujeres), H (hombres).

Los artículos que se tuvieron en cuenta para la selección fueron investigaciones vinculadas con la insatisfacción de la imagen corporal y su relación con la autoeficacia en el grupo etario equivalente, en que la edad osciló entre 8 y 19 años. De ellos, $60 \%$ incluye en su muestra jóvenes adolescentes con edades de 17 a 19 años. La mayoría de los estudios se desarrollaron en Latinoamérica, resaltando los países de México y Brasil; de igual modo, se realizaron estudios en otros países europeos y euroasiáticos (véase Tabla 1). 
Las variables más estudiadas fueron la percepción de la imagen corporal relacionada con la actividad física, con riesgo de trastorno de conductas alimentarias (TCA); de igual manera, otros estudios se relacionaron con prácticas conductuales en la satisfacción con la apariencia y la autopercepción y sus prácticas para corregirlas, así como la insatisfacción o distorsión en función del sexo y la percepción de salud, para un total de $75 \%$ de los trabajos. En el otro $25 \%$, se midieron la variable autoeficacia relacionada con el papel de las creencias, la preocupación por el IMC y la autoestima, la aptitud física y el riesgo alimenticio. En algunos casos se midió la motivación intrínseca y extrínseca como desmotivación independiente.

Cabe destacar que solo cinco de los estudios abordaron la relación entre autoeficacia e imagen corporal positiva o negativa, sin ocupar la variable de insatisfacción en las intervenciones. El 80\% de las investigaciones utilizaron instrumentos para evaluar la percepción de la imagen corporal satisfecha o insatisfecha, como el test de Siluetas de Stunkard, el IMC calculado a partir del peso y la talla autorreportados, los modelos anatómicos, la construcción del sí mismo, el Cuestionario Breve de Conductas Alimentarias de Riesgo, las mediciones antropométricas de talla y peso y, de forma cualitativa, los Cuestionarios de Autopercepción.

\section{DISCUSIÓN}

El objetivo del presente estudio fue analizar, por medio de una revisión sistemática, la relación entre la autoeficacia y la insatisfacción de la imagen corporal en adolescentes, hallando que en la mayoría de los artículos revisados la muestra de adolescentes tenía entre 9 y 19 años de edad, con una media de 17 (Da Silva y Hasselmann, 2019; Duno y Acosta, 2019; Escandón, Vargas, Herrera y Pérez, 2019; Franco, Díaz, F. y Bautista, 2019; Mendoza y Olalde, 2019; Meza y Pompa, 2018; Moffitt et al., 2018; Palacios y Ramírez, 2016; Peralta et al., 2016; Rossi et a1., 2020; Sánchez et al., 2019); otros autores incluyeron adolescentes con edades de entre 11 y 15 años, con una media de 13 años (Amaya et al., 2019; Bully et al., 2016; Cal- dera et al., 2019; De Pinho et al., 2019; Escolar et al., 2019; Flanagan y Perry, 2018; Ghahremani et al., 2018; Molina et al., 2018; Pellizzer, Waller y Wade, 2018; Platas et al., 2019; Rostami et al., 2017; Verdugo et al., 2020; Vurgun, 2015).

En relación con la insatisfacción de la imagen corporal, en la mayoría de los estudios se identifica al sexo femenino como predominante sobre el masculino; sin embargo, se obtuvieron resultados en los que los adolescentes de ambos sexos representaban un elevado porcentaje (Duno y Acosta, 2019), en los que la forma de percibir el propio cuerpo es diferente; así, el hombre desea mayor volumen muscular, mientras que las mujeres desean menos volumen y tener una silueta más delgada (Bully et al., 2016; De Pinho et al., 2019; Platas et al., 2019; Sánchez et al., 2019; Silva et al., 2019; Verdugo et al., 2020). Contrario a lo anterior, diversos autores señalan que la insatisfacción corporal solo se presenta en las mujeres, ya que en esas edades es cuando muestran conductas alimentarias de riesgo (Franco et al., 2019; Mendoza y Olalde, 2019; Vurgun, 2015).

Algunos autores detallan elementos que conducen a los adolescentes a presentar insatisfacción con su imagen corporal, ya que se encuentran en una etapa crítica para el crecimiento debido a las transformaciones físicas, sociales y psicológicas que en él ocurren (De Pinho et al., 2019). La edad es otro predictor directo de la insatisfacción para la interiorización de la figura ideal en ambos sexos (Hernández et al., 2016). Del mismo modo, en varios de dichos estudios se relaciona el peso corporal con el sexo (Escandón et al., 2019), elemento que corrobora la insatisfacción corporal por el aumento de IMC, esto es, las medidas de talla y peso que el adolescente desea, por lo que las mujeres quieren disminuir la silueta y los hombres aumentar el volumen muscular (Bully et al., 2016; De Pinho et al., 2019; Duno y Acosta, 2019; Mendoza y Olalde, 2019; Platas et al., 2019); otros adolescentes adoptan conductas alimentarias de riesgo debido a una práctica inadecuada para disminuir el peso (Franco et al., 2019).

Además, se observó que las influencias sociales tienen un considerable efecto en la percepción de las mujeres respecto a su imagen corporal, 
habiendo variables socioculturales que influyen de forma reveladora en la formación de una imagen corporal negativa, por lo que son motivos de preocupación tanto por el cuerpo, como por el malestar psicológico que este genera (Venegas y González, 2020).

También se encontró que la insatisfacción corporal negativa en los adolescentes se relaciona con varios comportamientos inadecuados significativos que pueden afectar su salud si no realizan las prácticas convenientes para enfrentar la situación. Autores como Flanagan y Perry (2018) plantean que la autoeficacia influye sobre la imagen corporal mediante la participación en prácticas de actividad física, así como en la salud, y como última instancia en aquellas situaciones sociales relacionadas con el peso corporal.

En cuanto a la relación entre autoeficacia e imagen corporal, hay diversos estudios que demuestran la efectividad de la capacidad de emprender y mantener comportamientos sanos al ejecutar acciones para tener estilos de vida saludable, como eliminar conductas alimentarias que pueden afectar la salud (Palacios y Ramírez, 2016; Pellizzer et al., 2018).

La búsqueda permitió hallar diferentes formas de abordaje de este tópico a partir de diferentes formas de evaluación y de estrategias de intervención para eliminar el proceso negativo de percepción de la imagen corporal. Como ya se evidenció, existe poca producción científica en este campo, por lo que es posible que haya una variedad de concepciones en cuanto a la terminología utilizada para analizar el fenómeno de la insatisfacción con la imagen corporal, misma que constituye el descontento del individuo con la forma de su cuerpo, o bien con ciertas partes del mismo. Los adolescentes son un grupo de población que se interesan particularmente en su imagen, y por lo general se encuentran insatisfechos por tener una percepción ideal o real negativa de su cuerpo, lo que llega a afectar su autoestima al haber una discrepancia respecto a cómo quieren ser (Escandón et al., 2019).

Varios estudios permiten confirmar los efectos positivos de utilizar una intervención con acciones de autoeficacia para invertir la percepción negativa de la imagen corporal en los adolescentes a través de fortalecer la satisfacción corporal, la autoestima, el autoconcepto y la motivación, para así fomentar su participación en actividades deportivas y ejecutar prácticas adecuadas para el mejoramiento de su salud, por lo que "es útil la aplicación de proyectos de promoción de hábitos saludables para educar mediante la búsqueda de un estilo de vida saludable y, con ello, incluir la variable de autoeficacia como elemento del proceso motivacional psicológico para tomar decisiones en situaciones positivas o negativas que beneficien la salud" (Del Río, Rodríguez, Rodríguez y Águila, 2018).

Este trabajo tiene cuando menos tres limitaciones. La primera es que aun cuando se consultaron las bases de datos de Scielo, Scopus, Dialnet, Ebsco y Pubmed en los idiomas español, inglés y portugués -lo cual proporciona una idea general de la realidad-, hay más artículos sobre el problema en otras bases de datos y en otros idiomas. Una segunda limitante es que algunos artículos no son de libre acceso, y la tercera es que se identifica en este estudio el sesgo de la publicación; es decir, cuando no se identifican los estudios que han sido publicados en revistas no indizadas, de circulación limitada o en la literatura gris.

Dentro de las aplicaciones prácticas se considera que los resultados de la presente investigación permitirán a quienes realicen estudios sobre la autoeficacia y su relación con la insatisfacción de la imagen corporal tener una base inicial acerca de los instrumentos más utilizados en la medición de dichos constructos.

Citación: Nápoles N., N., Blanco V., H. y Jurado G., P.J. (2022). La autoeficacia y su influencia en la insatisfacción de la imagen corporal: revisión sistemática. Psicología y Salud, 32(1), 57-70. https://doi.org/10.25009/pys.v32i1.2711. 


\section{REFERENCIAS}

Albertson, E., Neff, K. y Dill, K. (2015). Self-compassion and body dissatisfaction in women: A randomized controlled trial of a brief meditation intervention. Mindfulness, 6(3), 444-454. Doi: 10.1007/s12671-014-0277-3.

Amaya, A., Álvarez, G., Ortega, M. y Mancilla, J. (2019). Influencia de pares en preadolescentes y adolescentes: un predictorde la insatisfacción corporal y las conductas alimentarias anómalas. Revista Mexicana de Trastornos Alimentarios, 8(1), 31-39.

Arévalo, M. (2017). Actividad física, imagen corporal y clase social: un análisis a la literatura científica desde los modelos de determinantes sociales de la salud y determinación social de la salud. Tesis doctoral inédita. Bogotá: Universidad del Rosario.

Babativa, O.E. (2016). La posición socio económica en el campo de la actividad física y su relación con la imagen corporal: Aproximación desde el enfoque determinantes/determinación social de la salud. Tesis inédita de licenciatura. Rosario (Argentina): Universidad Nacional de Rosario.

Bandura, A. (1977). Social learning theory (v. 1). Englewood Cliffs, NJ: Prentice-Hall.

Bazán C., I. y Miño, R. (2015). La imagen corporal en los medios de comunicación masiva. Psicodebate. Psicología, Cultura y Sociedad, 15(1), 23-42.

Blanco, H., Díaz, A., Ornelas, M. y Mondaca, F. (2019). La autoeficacia en el cuidado de la salud en la predicción de la satisfacción con la vida. Revista Iberoamericana de Diagnóstico y Evaluación e Avaliação Psicológica, 3(52), 53-65.

Bonilla, P., Romero, L. y Cabrera, J. (2015). Calidad de vida, indicadores antropométricos y satisfacción corporal en un grupo de jóvenes colegiales. Retos. Nuevas Tendencias en Educación Física, Deporte y Recreación, 27, 62-66.

Bully, P., Elosua, P. y Jáuregui, A. (2016). Insatisfacción corporal en la adolescencia: Evolución en una década. Anales de Psicología, 28(1), 196-202.

Caldera J., F., Reynoso O., U., Nuño, D., Caldera I., A., Pérez, I. y Gómez C., A. (2019). Insatisfacción con la imagen corporal y personalidad en estudiantes de bachillerato de la región Altos Sur de Jalisco, México. Duazary, 16(1), 93-103. Doi: $10.21676 / 2389783 X .2534$.

Calvo J., M.C., Pimiento P., M.L. y Ríos P., J.V. (2019). Factores que influyen en la insatisfacción corporal de los adolescentes. Tesis inédita de licenciatura. Medellín (Colombia): Universidad Cooperativa de Colombia.

Cano, M. y Gianella, M. (2019). Factores de riesgo en problemas de conducta alimentaria e imagen corporal en adolescentes de una universidad de Lima. Tesis inédita de de Licenciatura. Lima: Universidad de Lima.

Castro, N. (2017). Re-conceptualización del constructo de imagen corporal desde una perspectiva multidisciplinar. Arbor, 192(781), a353. Doi: http://dx.doi.org/10.3989/arbor.2016.781n5010.

Cruzat, C., Díaz, F., Lizana, P. y Castro, A. (2016). Comparación por sexo en imagen corporal, síntomas psicopatológicos y conductas alimentarias en jóvenes entre 14 y 25 años. Revista Médica de Chile, 144(6), 743-750.

Da Silva A., M. y Hasselmann M., H. (2019). Violência familiar e insatisfaçao com a imagem corporal entre adolescentes do Programa Bolsa Família tratados numa unidade básica de saúde. Adolescencia e Saude, 16(2), 27-37.

De la Cruz, E., Arévalo, C. y Rojas M., P. (2018). Subestimación del índice de masa corporal a través de la autopercepción de la imagen corporal en sujetos con sobrepeso y obesidad. Archivos Venezolanos de Farmacologia y Terapéutica, 37(3), 235-240.

De Pinho, L., Santos, M., Ramos, R., Brito, R., De Olivera, C., Barbosa, D. y Prates, A. (2019). Percepção da imagem corporal e estado nutricional em adolescentes de escolas públicas. Revista Brasileira de Enfermagem, 72(2), 229-235. Doi: 10.1590/0034-7167-2018-0644.

Del Río, B., Rodríguez, M., Rodríguez K., L. y Águila, O. (2018). La autoeficacia docente: un reto en el accionar del profesor universitario de las ciencias médicas. Edumecentro, 10(2), 171-187.

Delgado, P., Caamaño, F., Osorio, A., Jerez, D., Fuentes, J., Levin, E. y Tapia, J. (2017). Imagen corporal y autoestima en niños según su estado nutricional y frecuencia de actividad física. Revista Chilena de Nutrición, 44(1), 12-18.

Díaz, M.C., Bilbao, G.M., Unikel, C., Escalante, E. y Parra, A. (2019). Relación entre estatus nutricional, insatisfacción corporal y conductas alimentarias de riesgo en estudiantes de nutrición. Revista Mexicana de Trastornos Alimentarios, 10(1), 53-65.

Duno, M. y Acosta, E. (2019). Percepción de la imagen corporal en adolescentes universitarios. Revista Chilena de Nutrición, 46(5), 545-553.

Escandón, N., Vargas J., F., Herrera A., C. y Pérez A., M. (2019). Imagen corporal en función de sexo y estado nutricional: Asociación con la construcción del sí mismo y de los otros. Revista Mexicana de Trastornos Alimentarios, 10(1), 32-41.

Escolar, M., Martínez, M., González, M., Medina, M., Mercado, E. y Lara, F. (2019). Factores de riesgo de trastornos de la conducta alimentaria entre universitarios: Estimación de vulnerabilidad por sexo y edad. Revista Mexicana de Trastornos Alimentarios, 8(2), 105-112. 
Estrada, F., Campero, L., Suárez, L., De la Vara, E. y González, G. (2017). Conocimientos sobre riesgo de embarazo y autoeficacia en hombres adolescentes: apoyo parental y factores escolares. Salud Pública de México, 59(5), 556-565. Doi: $10.21149 / 7959$.

Fernández, J., González, I., Contreras, O. y Cuevas, R. (2015). Relación entre imagen corporal y autoconcepto físico en mujeres adolescentes. Revista Latinoamericana de Psicología, 47(1), 25-33.

Flanagan, E. y Perry, A. (2018). Perception of physical fitness and exercise self-efficacy and its contribution to the relationship between body dissatisfaction and physical fitness in female minority children. International Journal of Environmental Research and Public Health, 15(6), 2-9. Doi: 10.3390/ijerph15061187.

Fraile, J., Tejero, C., Esteban, I. y Veiga, Ó. (2019). Asociación entre disfrute, autoeficacia motriz, actividad física y rendimiento académico en educación física. Retos, 2(32), 58-63.

Franco, K., Díaz, F. y Bautista, M.L. (2019). Estatus de peso, conductas alimentarias de riesgo e insatisfacción corporal en mujeres adolescentes y jóvenes. Archivos de Medicina, 19(2), 303-312. Doi: 10.30554/archmed.19.2.3283.2019.

Ghahremani, L., Hemmati, N., Kaveh, M. y Fararoei, M. (2018). Effects of an educational intervention targeting body image on self-esteem of Iranian high-school students: a quasi-experimental trial. Archives of Psychiatry and Psychotherapy, 1 , 59-66. Doi: 10.12740/APP/81549.

Gillen, M. y Lefkowitz, E. (2012). Gender and racial/ethnic differences in body image development among college students. Body Image, 9(1), 126-130. Doi: 10.1016/j.bodyim.2011.09.004.

Gómez, M., Gutiérrez, J., Llanos, C. y Babativa, H. (2017). El sentimiento de insatisfacción de la imagen corporal: un asunto a trabajar por medio de la educación física. Tesis inédita de licenciatura en Educación Física. Bogotá: Corporación Universitaria Minuto de Dios.

Guadarrama, R., Carrillo S., S., Márquez, O., Hernández J., C. y Veytia, M. (2014). Insatisfacción corporal e ideación suicida en adolescentes estudiantes del Estado de México. Revista Mexicana de Trastornos Alimentarios, 5(2), 98-106.

Gutiérrez, R. (2016). Asociación entre una agencia de autocuidado y autoeficacia percibida en personas que asisten a rehabilitación cardiaca. Tesis inédita de maestría. Bogotá: Universidad Nacional de Colombia.

Herencia A., M. (2017). Autoeficacia y síndrome de burnout en docentes del Colegio de Aplicación de la Universidad Nacional de Educación Enrique Guzmán y Valle. Tesis inédita de maestría. Lima: Universidad Nacional de Educación Enrique Guzmán y Valle.

Hernández, A., Díaz, J.M., Rayón, G., Luyando, M., López, M. y Guerrero, J.I. (2016). Edad, consciencia e interiorización del ideal corporal como predictores de insatisfacción y conductas alimentarias anómalas. Revista Colombiana de Psicología, 22(1), 121-133.

Horcajo, L., Quiles, Y. y Quiles, M.J. (2019). Aplicación de la terapia centrada en la compasión en pacientes con un trastorno de la conducta alimentaria: un estudio piloto. Psicología Conductual, 27(2), 295-309.

Jiménez, P., Cruz, A. y Bacardí, M. (2017). Insatisfacción con la imagen corporal en niños y adolescentes: revisión sistemática. Nutrición Hospitalaria, 34(2), 479-489.

León M., P., González, I., Fernández J., G. y Contreras, O. (2018). Percepción del tamaño corporal e insatisfacción en niños de 3 a 6 años: una revisión sistemática. Anales de Psicología, 34(1), 173-183.

López G,. F., Díaz, A. y Smith, L. (2018). Análisis de imagen corporal y obesidad mediante las siluetas de Stunkard en niños y adolescentes españoles de 3 a 18 años. Anales de Psicología, 34(1), 167-172.

Manrique R., B., Manrique R., L., Vallejo K., M., Manrique C., C., Santamaría A., M. y Pincay A., G. (2018). Trastornos corporales en adolescentes e influencia de los medios de comunicación. Revista Cubana de Investigaciones Biomédicas, $37(4), 1-11$.

Mendoza, M. y Olalde, G. (2019). Autopercepción de la imagen corporal y conductas alimentarias de riesgo en estudiantes universitarios de Medicina en Xalapa, Veracruz, México (2014). Revista de Ciencias de la Salud, 17(1), 34-52.

Meza, C. y Pompa, E. (2018). Distorsión e insatisfacción con la imagen corporal en una muestra de mexicanos. Revista de Psicología y Ciencias del Comportamiento de la Unidad Académica de Ciencias Jurídicas y Sociales, 9(2), 120-131.

Moffitt, R., Neumann, D. y Williamson, S. (2018). Comparing the efficacy of a brief self-esteem and self-compassion intervention for state body dissatisfaction and self-improvement motivation. Body Image, 27, 67-76.

Molina D., C., Gonçalves, S., Guimaraes, G. y Aerts, D. (2018). Body image satisfaction and subjective wellbeing among ninth-grade students attending state schools in Canoas, Brazil. Ciencia \& Saude Coletiva, 23(12), 4289-4298. Doi: $10.1590 / 1413-812320182312.14742016$.

Moral, J.E., Agraso, A.D., Ramos, A.J., Jiménez, A. y Jiménez E., A. (2020). The influence of physical activity, diet, weight status and substance abuse on students' self-perceived health. International Journal of Environmental Research and Public Health, 17(4), 1387. Doi: 10.3390/ijerph17041387. 
Moreno, P. y Bueno, J.A. (2019). Evaluación de la percepción de autoeficacia en pacientes de oncología infantil. Psicooncologia, 16(2), 387-403. Doi: 10.5209/psic.65598.

Ojeda, F. (2016). Historia de una caida. Estudio de caso sobre la trayectoria sociobiográfica de una adolescente suicida. Tesis inédita de maestría. Santiago de Chile: Universidad de Chile.

Olivos, M.A. (2018). Autoeficacia escolar y rendimiento escolar de los estudiantes de la Institución Educativa "Víctor Raúl Haya de la Torre" Agomarca-Bambamarca, 2018. Tesis inédita de maestría. Lima: Universidad César Vallejo.

Orcasita L., T., Mosquera J., A. y Carrillo, T. (2018). Autoconcepto, autoeficacia y conductas sexuales de riesgo en adolescentes. Informes Psicológicos, 18(2), 141-168. Doi: 10.18566/infpsic.v18n2a08.

Pacheco, B.M., Lozano, J.L. y González, N. (2018). Diagnóstico de utilización de redes sociales: factor de riesgo para el adolescente. Revista Iberoamericana para la Investigación y el Desarrollo Educativo, 8(16), 53-72.

Palacios, J.R. y Ramírez, V. (2016). Estudio comparativo de la autoeficacia saludable en las conductas alimenticias de riesgo en jóvenes. Psicología Iberoamericana, 24(2), 17-25.

Pallo, C.A. (2017). Relación entre la frecuencia de consumo de frutas y verduras, actividad fisica con el nivel de estrés académico en estudiantes del Instituto Tecnológico Superior Vida Nueva, Quito-Ecuador, 2016. Tesis inédita de licenciatura. Lima: Universidad Peruana Unión.

Pellizzer, M., Waller, G. y Wade, T. (2018). Body image flexibility: A predictor and moderator of outcome in transdiagnostic outpatient eating disorder treatment. International Journal of Eating Disorders, 51(4), 368-372.

Peralta, R.E. y Quintana S., M.R. (2016). Autopercepción de la imagen corporal y prácticas para corregirla en adolescentes de una institución educativa, Lima-Perú. Anales de la Facultad de Medicina, 77(2), 117-122.

Pérez F., M.C., Molero J., M.M., Barragán M., A.B., Martos M., Á., Simón M., M.M. y Gázquez L., J.J. (2018). Autoeficacia y engagement en estudiantes de Ciencias de la Salud y su relación con la autoestima. Publicaciones de la Facultad de Educación y Humanidades del Campus de Melilla, 48(1), 193-210. Doi: 10.30827/publicaciones.v48i1.7323.

Pérez L., B. y Trujillo L., F. (2018). Efectividad de un programa de autocompasión basado en ACT en un contexto de educación inclusiva. Tesis inédita de licenciatura. Bogotá: Corporación Universitaria Minuto de Dios.

Platas, R., Gómez, G. y Pineda, G. (2019). Subestimación del peso corporal en escolares mexicanos. Psicología y Salud, 29(2), 269-277.

Pruzinsky, T. y Cash, T. (1990). Body images: Development, deviance, and change. Washington, DC: The Guilford Press.

Quiliano, M. R. (2015). Conductas saludables para mejorar la calidad de vida de la mujer climatérica de la zona urbana del distrito de Huancayo. Tesis doctoral inédita. Huancayo (Perú): Universidad Nacional del Centro de Perú.

Ramírez, M.J. (2017). Imagen corporal, satisfacción corporal, autoeficacias especificas y conductas de salud y riesgo para la mejora de la imagen corporal. Tesis doctoral inédita. Granada (España): Universdad de Granada.

Reyna, J.E. (2019). Insatisfacción con la imagen corporal y agresividad premeditada e impulsiva en adolescentes de la ciudad de Trujillo, 2019. Tesis inédita de licenciatura. Trujillo (Perú): Universidad Cesar Vallejo.

Rossi, T., Trevisol, A., Dos Santos, D., Dapieve, N. y Von, J. (2020). Autoeficacia general percibida y motivación para aprender en adolescentes de educación media. Acta Colombiana de Psicología, 23(1), 254-263.

Rostami, S., Fallahi, A., Pashaei, T. y Roshani, D. (2017). Association of Trans-Theoretical Model (TTM) based exercise behavior change with body image evaluation among female Iranian students. International Journal of Pediatrics, 5(3), 4613-4623. Doi: 10.22038/ijp.2017.21152.1776.

Sánchez, S., López, G., Sgroi, M. y Díaz, A. (2019). Imagen corporal y obesidad mediante las siluetas de Stunkard en adolescentes italianos de 14 a 21 años. Journal of Sport \& Health Research, 11(2), 199-210.

Sierra, M. (2017). Relación entre satisfacción de la imagen corporal y la autoestima en estudiantes de Medicina y Enfermería. UNSA-Arequipa 2016. Tesis inédita de maestría. Arequipa (Perú): Universidad Nacional de San Agustin de Arequipa.

Silva, C., Fernández, N. y Rodríguez, N. (2019). Depresión, ansiedad y autoeficacia para bajar de peso en mujeres en tratamiento nutricional. Psicología y Salud, 29(1), 41-49.

Simón, C. (2017). El estigma social y mediático de la salud mental. Tesis inédita de licenciatura. Santa Cruz de Tenerife (España): Universidad de la Laguna.

Solano, N., Cano, A., Blanco, H. y Fernández, R. (2017). Datos psicométricos de la versión abreviada del cuestionario Imagen: evaluación de la insatisfacción corporal. Nutrición Hospitalaria, 34(4), 952-960.

Venegas K., S. (2019). Efecto de una intervención psicológica sobre la imagen corporal en mujeres adolescentes y adultas jóvenes. Tesis doctoral inédita. Monterrey (México): Universidad Autónoma de Nuevo León.

Venegas, K. y González, M. (2020). Influencias sociales en un modelo de insatisfacción corporal, preocupación por el peso y malestar corporal en mujeres mexicanas. Acta Colombiana de Psicología, 23(1), 7-17. 
Verdugo, G., Armas, E., Baños, R., Moncada, J. y Rentería, I. (2020). Distorsión de la imagen corporal y trastornos alimentarios en adolescentes gimnastas respecto a un grupo control de adolescentes no gimnastas con un IMC. Retos, 37(37), $297-302$.

Vurgun, N. (2015). Effects of regular aerobic exercise on physical characteristics, body image satisfaction and self-efficacy of middle-aged women. South African Journal for Research in Sport, Physical Education and Recreation, $37(1), 151-163$.

Yadav, V.P. (2017). Understanding the body image of adolescents: A psychological perspective. International Journal of Applied Research, 3(6), 588-594. 